Comparative and Functional Genomics

Comp Funct Genom 2005; 6: 25I-255.

Published online in Wiley InterScience (www.interscience.wiley.com). DOI: 10.1002/cfg.479

\title{
Conference Review
}

\section{Swine Genome Sequencing Consortium (SGSC): a strategic roadmap for sequencing the pig genome}

\author{
Lawrence B. Schook ${ }^{1,2 *}$, Jonathan E. Beever ${ }^{1,2}$, Jane Rogers ${ }^{3}$, Sean Humphray ${ }^{3}$, Alan Archibald ${ }^{4}$, \\ Patrick Chardon" ${ }^{5}$, Denis Milan ${ }^{6}$, Gary Rohrer ${ }^{7}$ and Kellye Eversole ${ }^{8}$ \\ I Institute for Genomic Biology, University of Illinois, Urbana, IL, USA \\ ${ }^{2}$ Department of Animal Sciences, University of Illinois, Urbana, IL, USA \\ ${ }^{3}$ The Wellcome Trust Sanger Institute, Hinxton, UK \\ ${ }^{4}$ Roslin Institute, Edinburgh, UK \\ 5 INRA-CEA, Jouy-en-Josas, France \\ 'INRA-Toulouse, France \\ ${ }^{7}$ Agricultural Research Senvice, Clay Center, NE, USA \\ ${ }^{8}$ The Alliance for Animal Genomics, Bethesda, MD, USA
}

*Correspondence to: Lawrence B. Schook,

Departments of Animal Sciences and Veterinary Pathobiology,

University of Illinois, 382 Edward R. Madigan Laboratory, I20I W.

Gregory Dr., Urbana, IL 61801, USA.

E-mail: schook@uiuc.edu
Received: 22 February 2005

Revised: 17 March 2005

Accepted: 18 March 2005

\begin{abstract}
The Swine Genome Sequencing Consortium (SGSC) was formed in September 2003 by academic, government and industry representatives to provide international coordination for sequencing the pig genome. The SGSC's mission is to advance biomedical research for animal production and health by the development of DNAbased tools and products resulting from the sequencing of the swine genome. During the past 2 years, the SGSC has met bi-annually to develop a strategic roadmap for creating the required scientific resources, to integrate existing physical maps, and to create a sequencing strategy that captured international participation and a broad funding base. During the past year, SGSC members have integrated their respective physical mapping data with the goal of creating a minimal tiling path (MTP) that will be used as the sequencing template. During the recent Plant and Animal Genome meeting (January 16, 2005 San Diego, CA), presentations demonstrated that a human-pig comparative map has been completed, BAC fingerprint contigs (FPC) for each of the autosomes and $X$ chromosome have been constructed and that BAC end-sequencing has permitted, through BLAST analysis and RH-mapping, anchoring of the contigs. Thus, significant progress has been made towards the creation of a MTP. In addition, whole-genome (WG) shotgun libraries have been constructed and are currently being sequenced in various laboratories around the globe. Thus, a hybrid sequencing approach in which $3 x$ coverage of BACs comprising the MTP and $3 x$ of the WG-shotgun libraries will be used to develop a draft $6 x$ coverage of the pig genome. Copyright $(\underset{2005}{20 h n}$ Wiley \& Sons, Ltd.
\end{abstract}

Keywords: genomics; pig genome; porcine; physical maps; sequencing consortium; whole-genome sequencing

\section{Background and discussion}

The pig genome is of similar size, complexity and chromosomal organization $(2 n=38$, including meta- and acrocentric chromosomes) as the human genome. Over the past decade tremendous progress has been made in mapping and characterizing the swine genome. Currently, moderate- to highresolution genetic linkage maps containing highly polymorphic loci (Type II) have been produced using independent mapping populations (Rohrer et al., 1996; Ellegren et al., 1994; Archibald et al., 
1995). Additionally, physical mapping methods such as somatic cell hybrid analysis (Rettenberger et al., 1994; Yerle et al., 1996), in situ hybridization, and ZOO-FISH (Chowdhary et al., 1996; Fronicke et al., 1996; Goureau et al., 1996) have been employed to enrich the Type I marker map, and to perform comparative analysis with maprich species such as the human and mouse. To date, $>3000$ mapped loci are catalogued for the pig genome (http://www.thearkdb.org). Recently, whole-genome radiation hybrid (WG-RH) panels (7500 and $12500 \mathrm{rad}$ ) have been generated for swine (Hawken et al., 1999; Yerle et al., 2002), resulting in yet another rapid increase in the number of expressed sequences being mapped, facilitating comparative mapping with other species (Rinke et al., 2002). The swine genomics community has also acquired access to resources such as bacterial artificial chromosome (BAC) libraries (Fahrenkrug et al., 2001; Anderson et al., 2000) that provide approximately $35 \times$ coverage of the swine genome. These BAC resources have facilitated the production of high-resolution physical maps in specific chromosomal regions (Rogel-Gaillard et al., 1999; Milan et al., 2000) and support the construction of sequence-ready mapping resources for the porcine genome.

Comparative maps have indicated that the porcine and human genomes are more similarly organized than when either is compared to the mouse (Thomas et al., 2003). The mean length of conserved syntenic segments between human and pig is approximately twice as long as the average length of conserved syntenic segments between human and mouse (Ellergren et al., 1994; Rettenberger et al., 1995). Furthermore, the organizational similarities between the human and porcine genomes are reflected in similarities at the nucleotide level. In more than 600 comparisons of non-coding DNAs aligned by orthologous exonic sequences on human chromosome 7, pig (and cow, cat and dog) sequences consistently grouped closer to human and non-human primate sequences than did rodent (mouse and rat) sequences (Thomas et al., 2003). Furthermore, the rodent genomes are evolving at a different (faster) rate than other representative genomes. For these reasons it is necessary to produce the genomic sequence for eutherian mammals outside the primate and rodent lineages in order to better assemble and annotate the human sequence. During the Plant and Animal
Genome meeting, it was reported that a $1.0 \mathrm{Mb}$ human-pig comparative map has been completed (Meyers et al., 2005). This map will provide the basis for creating a MTP that will be used as the template for genome sequencing.

\section{Harvesting genomic information}

The porcine research community has a long history in quantitative genetics, and more recently in genomics research. The genetic contribution of many polygenic traits in pigs is well documented, and this knowledge has provided the basis for the identification and mapping of a growing number of quantitative trait loci (QTL) (Andersson et al., 1994; Milan et al., 2000; Rohrer et al., 1999; Wilkie et al., 2000; Paszek et al., 2000; Malek et al., 2001a,b; Nezer et al., 2002). These maps have been used to identify chromosomal regions that influence quantitative traits affecting growth, body composition, reproduction and immune response (Bidanel and Rothschild, 2002). The quantitative trait loci defined in these studies often span 20-40 centiMorgans (cM) and perhaps correspond to about 20-40 Mbp of DNA. These initial scans for the gene(s) controlling the phenotype of interest generally only reduce the search space to $1-2 \%$ of the genome, perhaps to 200-400 positional candidate genes. Locating the gene(s) responsible and identifying the causal molecular genetic variation is a major challenge. Nevertheless, there have been some striking successes in achieving this goal in pigs, to which some of the co-authors have contributed.

The only limitation to performing direct genetic experiments and identifying genes underlying these traits is the lack of a complete genome sequence. Selection experiments, heterosis studies and breed comparisons have all been used in porcine genetic studies. Many populations have been used to map genes to large chromosomal regions but positional mapping of causal genes has been difficult. Sequencing the porcine genome and generating 100000 SNPs will provide additional polymorphic markers and positional candidate genes based on the human and mouse map. Large populations with designed matings can be used to positionally map genes. The populations can be generated by natural reproduction, artificial insemination or assisted reproductive technologies. Clones can also be generated from fibroblasts or stem cells and 
cryopreserved. This technology provides the opportunity for knock-out or knock-in experiments in an animal other than the mouse. Interspecies porcine hybrids are easily produced and are very valuable for knock-out/knock-in experiments and studying genomic imprinting (Andersson et al., 1994).

\section{Justification for sequence information}

A CREES-USDA workshop during the summer of 2002, The Allerton III Conference ('Beyond Livestock Genomics') was designed to bring together leading investigators from broad disciplines (physiology, reproduction, animal health, nutrition and genetics) to begin to develop a plan for full utilization of genomic information to promote animal health and production (Hamernik et al., 2003). In February 2002, the National Academy of Sciences organized a public workshop, 'Exploring Horizons for Domestic Animal Genomics', to identify research goals and funding needs. Subsequent discussion identified a growing need to have a broader context for discussion to ensure full utilization of the genomic information and tools in support of animal research. Thus, the Allerton III Conference provided a venue for discussion of how genome sequences could be harvested to support the broader animal agricultural community, while contributing to life science discovery. The objectives of the Allerton III Conference included: (a) identification of genomic and bioinformatic tools and reagents required to exploit information from the human genome initiative; (b) discussion of needs and opportunities for full implementation of genomic capabilities by related disciplines; and (c) identification of needs and opportunities to ensure full technology transfer and commercialization (Hamernik et al., 2003).

\section{The Swine Genome Sequencing Consortium (SGSC)}

In September 2003, interested researchers convened at INRA-Jouy-en-Josas to establish the SGSC for facilitation and coordination of international efforts toward obtaining the complete porcine genome sequence. A coordinated international effort was initiated to develop a porcine BAC map with two BAC libraries (RPCI-44 and CHORI-242) made by Pieter J. de Jong, one library made at the Roslin Institute (Anderson et al., 2000), and a library produced at
INRA (Rogel-Gaillard et al., 1999). Through the exchange of BAC clones, data has been merged to permit a comprehensive analysis. INRA has screened more than 1000 BACs from this library for known genes and markers and has mapped them on genetic and RH maps. INRA is sharing this set of BACs to facilitate anchoring of contigs. Sequencing the ends of all fingerprinted BAC clones has also been conducted. The current status of the fingerprint contig (FPC) was discussed at the PAG 2005 meeting (Humphray et al., 2005; see Table 1). The final product, which is scheduled for completion in July 2005, will represent $20 \times$ coverage of the porcine genome.

During the past year, significant allocation of resources has occurred with respect to positioning the porcine genome sequencing initiative. This has included the development of a whole genome porcine BAC fingerprint with complete BAC endsequencing. Thus, to date, the SGSC has completed sequencing of over 500000 BAC ends (see Table 2), which represents over $13 \%$ sequence coverage of the pig genome (Humphray et al., 2005).

Table I. FPC Database (Wellcome Trust Sanger Institute)

\begin{tabular}{lcc}
\hline Library & Fingerprints & Complexity $^{\mathbf{c}}$ \\
\hline CHORI-242 & $103762^{\mathrm{a}}$ & $6.3 \times$ \\
PigE BAC (Roslin) & $73971^{\mathrm{a}}$ & $4.0 \times$ \\
RPCI-44 & $61104^{\mathrm{b}}$ & $4.0 \times$ \\
INRA & $28478^{\mathrm{a}, \mathrm{b}}$ & $1.0 \times$ \\
TOTAL & 267826 & $15.3 \times$ \\
\hline
\end{tabular}

a Wellcome Trust Sanger Institute: http://www.sanger.ac.ak/ Projects/S_scrofal

b University of Illinois at Urbana-Champaign.

c Based on $2.7 \mathrm{~Gb}$ genome size.

Table 2. BAC end sequencing results

\begin{tabular}{lcccc}
\hline Library & $\begin{array}{c}\text { Passed } \\
\text { reads }\end{array}$ & $\begin{array}{c}\text { Paired } \\
\text { ends (\%) }\end{array}$ & $\begin{array}{c}\text { Average } \\
\text { GC (\%) }\end{array}$ & $\begin{array}{c}\text { Average } \\
\text { length (bp) }\end{array}$ \\
\hline CHORI-242a,b $^{\mathrm{a}}$ & 276758 & 92 & 41 & 698 \\
PigE BAC $^{\mathrm{a}}$ & 145110 & 93 & 42 & 700 \\
RPCl-44 $^{\mathrm{b}}$ & 71847 & 87 & 40 & 521 \\
INRA $^{c}$ & 64102 & 94 & 42 & 613 \\
Total & 557898 & BACs representing 13\% of pig genome \\
\hline
\end{tabular}

a Wellcome Trust Sanger.

b University of Illinois at Urbana-Champaign.

c INRA. 


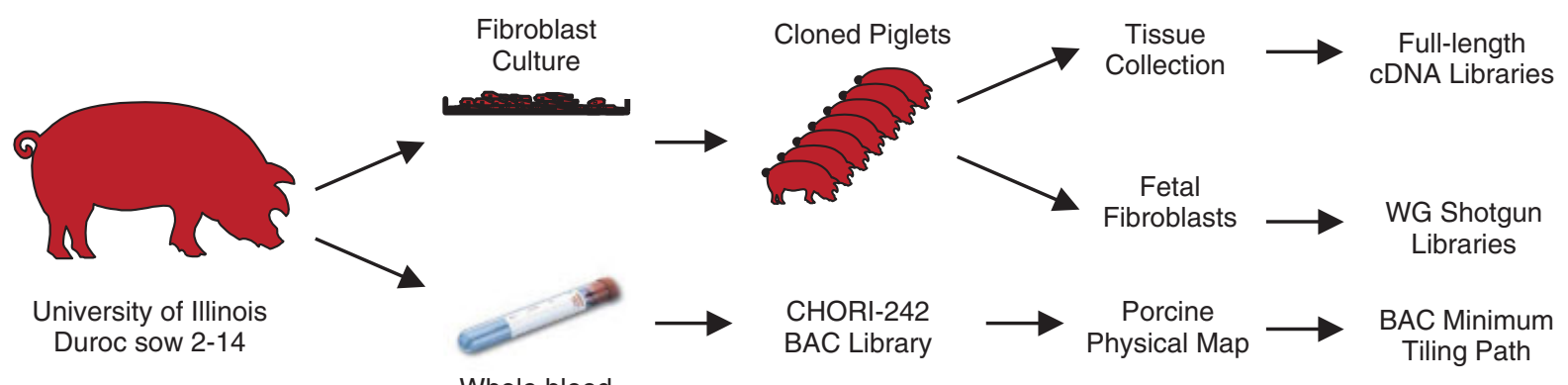

Figure I. Schema for the production of autologous reagents for sequencing the porcine genome. Duroc sow 2-14 was selected as the donor of the DNA used to create the CHORI-242 BAC library. She has been 'immortalized' by the establishment of fibroblast cell cultures that have been successfully used for nuclear transfer. Fetal fibroblast cultures from cloned piglets have been transferred to the Sanger Institute and genomic DNA from these cell lines has been used to create whole-genome shotgun libraries. Tissues from embryos at various stages of gestation have also been collected for use in the construction of full-length cDNA libraries

\section{The sequencing template}

The majority of clones that have been fingerprinted and end-sequenced have come from the CHORI242 BAC library. This library was constructed from a single female pig that was raised at the University of Illinois (Figure 1). To facilitate sequence assembly, efforts will be made to select as many CHORI242 clones as possible for the BAC minimum tiling path. Additionally, the WGS libraries will be made from autologous DNA to further enhance sequence assembly between WGS reads and those from the BAC skim. Full-length cDNA libraries will also be constructed from tissues belonging to the original sow or her clones, providing autologous sequence for gene annotation.

\section{Strategy for genomic sequencing}

The strategy that we espouse for sequencing the pig genome combines the whole-genome shotgun (WGS) approach with skim sequencing of BAC clones selected to represent a minimum tiling path through the pig genome (Engler et al., 2003; She et al., 2004). We propose that a draft sequence of the pig genome with $6-7 \times$ genome coverage be produced by this hybrid approach. A draft sequence does not provide complete coverage of the entire genome; indeed, there are still gaps in the current 'finished' human genome sequence. One of the key strengths of the hybrid approach is that the resources (BAC clones) will be in place for targeted sequence closure in regions of interest. An important difference between the application of this approach to the pig genome and its use for other species to date is that the porcine fingerprint map and $\mathrm{BAC}$ end sequence information will be completed before the sequencing project starts. Thus, it should be possible to determine a BAC tiling path from these two datasets, identifying a set of BACs with minimal overlap at the outset of the sequencing project. Current calculations predict that at most 25000 BACs will need to be sequence-skimmed, since the human genome is approximately $2.9 \mathrm{~GB}$ and the pig genome is approximately $2.6 \mathrm{~GB}$. This calculation is also supported by the increased size of the BAC inserts from $150 \mathrm{~kb}$ to a range of $160-180 \mathrm{~kb}$, thus reducing the number of BACs to be sequence-skimmed. The project will then sequence $3 \times$ coverage and the remaining 3-4x coverage will come from wholegenome sequencing of $3 \mathrm{~kb}, 10 \mathrm{~kb}$ and $50 \mathrm{~kb}$ libraries. The sequence will be released into public databases as it is generated, and sequence traces will be deposited in the trace repositories hosted at NCBI and EBI. Sequence assemblies $>2 \mathrm{~kb}$ will be deposited in the HTGS databases at NCBI and EMBL. It is anticipated that after the first year of sequencing, a draft $3 \times$ assembly of the genome will be released into public databases.

\section{Acknowledgements}

This work was partially supported by grants from the USDA-National Research Initiative (2002-35205-12712), the USDA Cooperative State Research Service (AG200234480-11828) and the USDA Agricultural Research Service (Agreement No. 58-5438-2-313). The authors wish 
to recognize the support of the College of Agricultural, Environmental and Consumer Sciences, University of Illinois. The SGSC also wishes to recognize the leadership provided by USDA Undersecretary J. Jen (in promoting animal genomics) and the CSREES (C. Hefferan, A. Palamiso, M. Poth, D. Hamernik, M. Qureshi, P. Brayton) and ARS (R. Green and S. Kappes) administration, and the Alliance for Animal Genome Research (R. Wyse and K. Eversole). Finally, support and assistance from M. Boggess of the NPB is acknowledged. Also, support from the UK BBSRC, Defra, Sygen and Roslin Institute is acknowledged.

\section{References}

Anderson SI, Lopez-Corrales NL, Gorick B, Archibald AL. 2000. A large fragment porcine genomic library resource in a BAC vector. Mamm Genome 11: 811-814.

Andersson L, Haley CS, Ellegren H, et al. 1994. Genetic mapping of quantitative trait loci for growth and fatness in pigs. Science 263: $1771-1774$.

Archibald AL, Haley CS, Brown JF, et al. 1995. The PiGMaP Consortium linkage map of the pig (Sus scrofa). Mamm Genome 6: $157-175$.

Bidanel JP, Rothschild MF. 2002. Current status of quantitative trait loci mapping in pigs. Pig News Inform 23: N39-N54.

Chowdhary BP, Raudsepp T, Fronicke L, Scherthan H. 1998. Emerging patterns of comparative genome organization in some mammalian species as revealed by ZOO-FISH. Genome Res $\mathbf{8}$ : $577-589$.

Ellergren H, Chowdhary BP, Johansson M, et al. 1994. A primary linkage map of the porcine genome reveals a low rate of genetic recombination. Genetics 137: 1089-1100.

Engler FW, Hatfield J, Nelson W, Soderlund CA. 2003. Locating sequence on FPC maps and selecting a minimal tiling path. Genome Res 13: 2152-2163.

Fahrenkrug SC, Rohrer GA, Freking BA, et al. 2001. A porcine BAC library with tenfold genome coverage: a resource for physical and genetic map integration. Mamm Genome 12: $472-474$.

Goureau A, Yerle M, Schmitz A, et al. 1996. Human and porcine correspondence of chromosome segments using bidirectional chromosome painting. Genomics 36: 252-262.

Gregory SG, Sekhorn M, Schein J, et al. 2002. A physical map of the mouse genome. Nature 418: 743-749.

Hamernik DL, Lewin HA, Schook LB. 2003. Allerton III. Beyond Livestock Genomics. Anim Biotech 14: 77-82.

Hawken RJ, Murtaugh J, Flickinger GH, et al. 1999. A first generation porcine whole-genome radiation hybrid map. Mamm Genome 10: 824-830.

Humphray SJ, Clark RC, Beever J, et al. 2005. An integrated physical map of the porcine genome. Proceedings of the Plant and Animal Genome XIII Conference, San Diego, CA: abstr P559; 210.

Malek M, Dekkers JCM, Lee HK, Baas TJ, Rothschild MF. 2001a. A molecular genome scan analysis to identify chromosomal region influencing economic traits in the pig. I. Growth and body composition. Mamm Genome 12: 630-636.

Malek M, Dekkers JCM, Lee HK, et al. 2001b. A molecular genome scan analysis to identify chromosomal regions influencing economic traits in the pig. II. Meat and muscle composition. Mamm Genome 12: 630-636.

Maruyama K, Sugano S. 1994. Oligo-capping: a simple method to replace the cap structure of eukaryotic mRNAs with oligoribonucleotides. Gene 138: 171-174.

Meyers SN, Rogatcheva MB, Yerle M, et al. 2005. PiggyBACing the human genome: II. A high resolution, physicallyanchored, comparative map of the porcine autosomes. Genomics (in press).

Milan D, Jeon TT, Looft C, et al. 2000. A mutation in PRKAG3 associated with excess glycogen content in pig skeletal muscle. Science 288: 1248-1251.

Nezer C, Moreau L, Wagenaar D, Georges M. 2002. Results of a whole-genome scan targeting QTL for growth and carcass traits in a Pietrain X Large White intercross. Genet Select Evol 34: 371-387.

Paszek AA, Wilkie PJ, Flickinger GH, et al. 1999. Interval mapping of growth in divergent swine cross. Mamm Genome 10(2): 117-122.

Rettenberger G, Klett C, Zecher U, et al. 1995. Visualization of the conservation of synteny between humans and pigs by heterologous chromosomal painting. Genomics 26: 372-378.

Rink A, Santchi EM, Eyer KM, et al. 2002. A first-generation EST RH comparative map of the porcine and human genome. Mamm Genome 13: 578-587.

Rohrer GA, Alexander LJ, Hu Z, et al. 1996. A comprehensive map of the porcine genome. Genome Res 6: 371-391.

Rohrer GA, Ford JJ, Wise TH, Vallet JL, Christenson RK. 1999. Identification of quantitative trait loci affecting female reproduction traits in a multigeneration Meishan-White composite swine population. J Anim Sci 77: 1385-1391.

Rogel-Gaillard C, Bourgeaux N, Billaut A, Vaiman M, Chardon P. 1999. Construction of a swine BAC library: application to the characterization and mapping of porcine type $\mathrm{C}$ endoviral elements. Cytogenet Cell Genet 85: 205-211.

She XZ, Jiang RA, Clark G, et al. 2004. Shotgun sequence assembly and recent segmental duplications within the human genome. Nature 431: 927-930.

Thomas JW, Touchman JW, Blakesley RW, et al. 2003. Comparative analyses of multi-species sequences from targeted genomic regions. Nature 424: 788-793.

Wilkie PJ, Paszek AA, Beattie CW, et al. 1999. A genomic scan of porcine reproductive traits reveals a possible quantitative trait loci (QTL) for number of corpora lutea. Mamm Genome 10: 573-578.

Yerle M, Echard G, Robic A, et al. 1996. A somatic cell hybrid panel for pig regional gene mapping characterized by molecular cytogenetics. Cytogenet Cell Genet 73: 194-202.

Yerle M, Pinton P, Delcros C, et al. 2002. Generation and characterization of a 12000-rad radiation hybrid panel for fine mapping in pig. Cytogenet Genome Res 97: 219-228. 

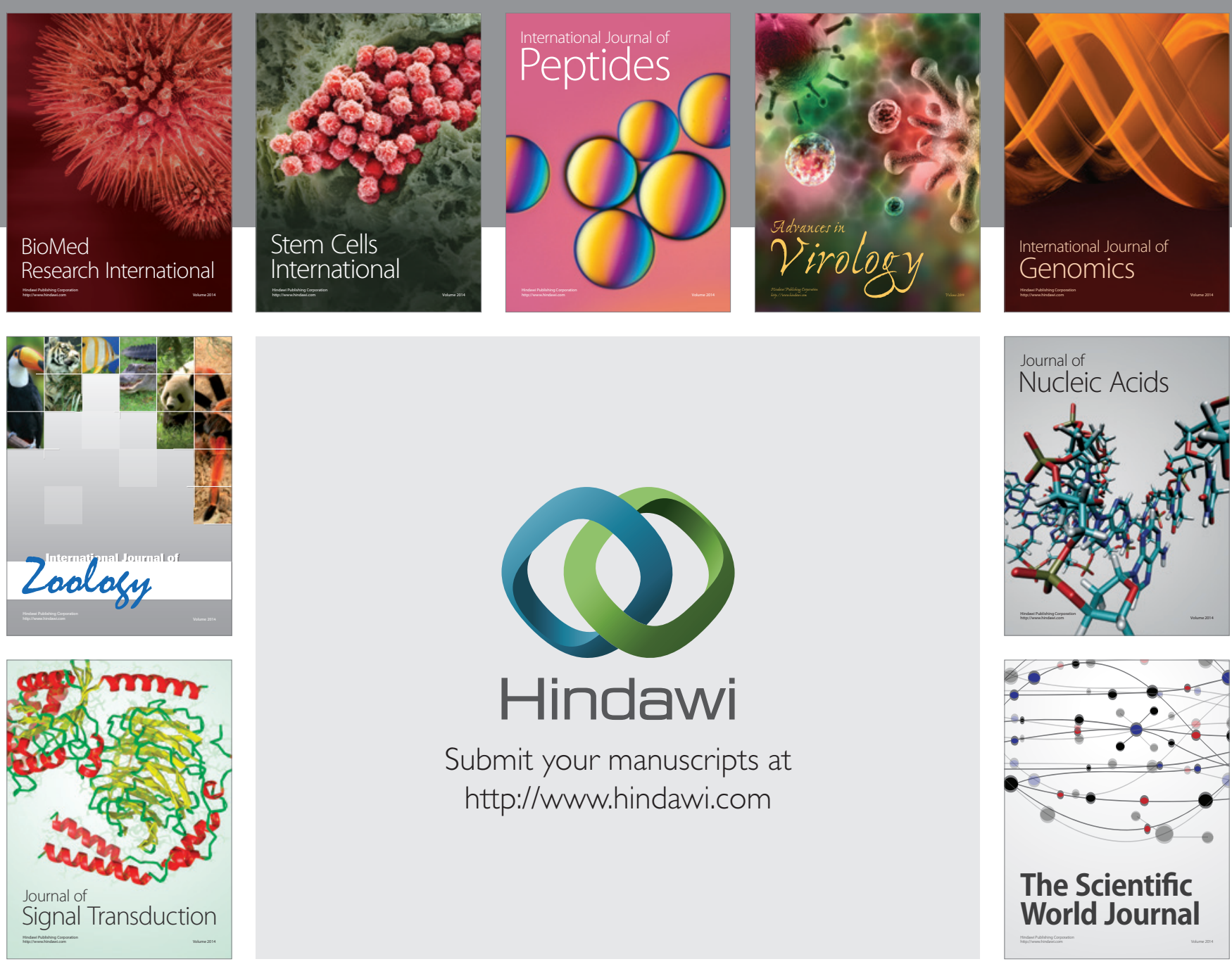

Submit your manuscripts at

http://www.hindawi.com
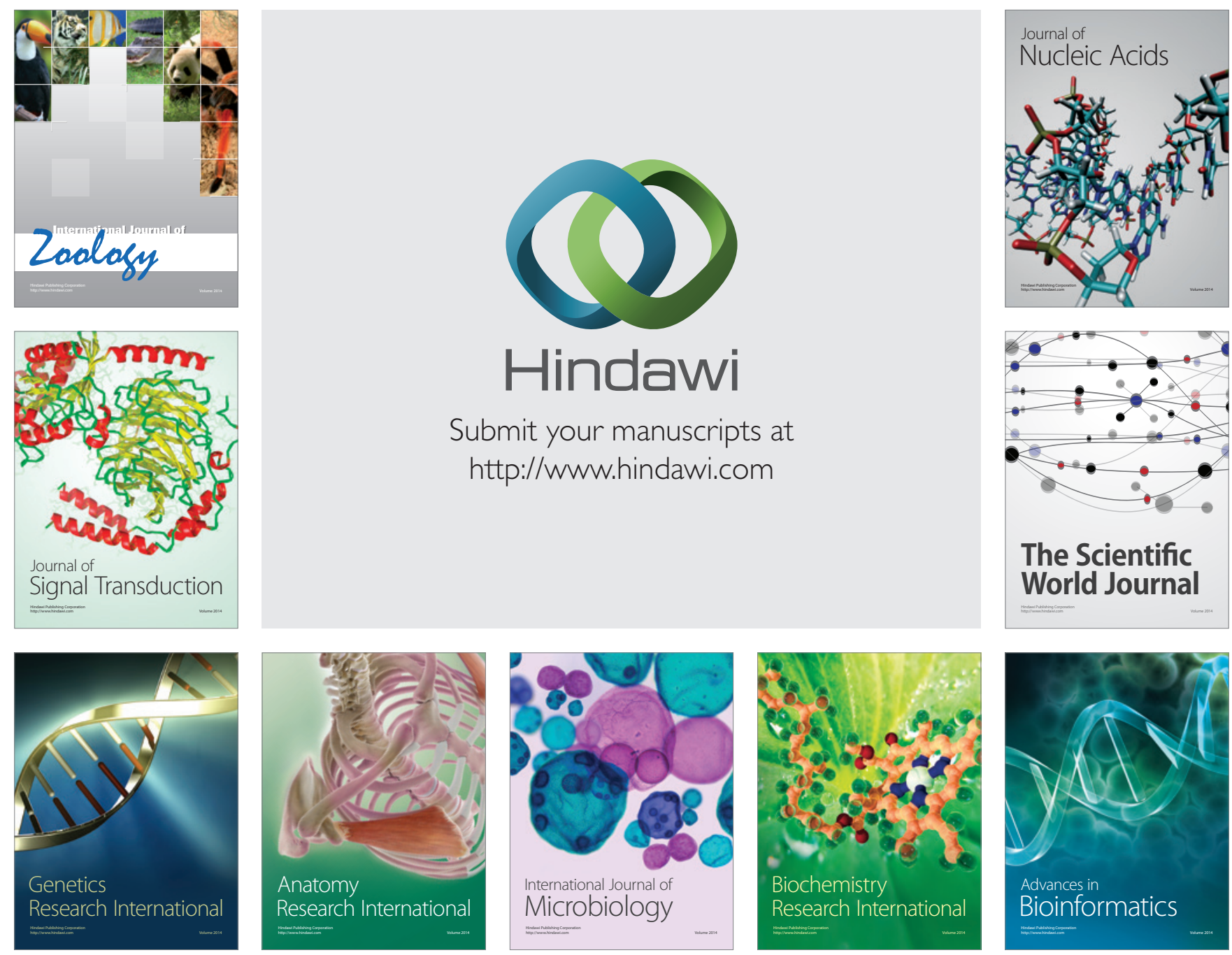

The Scientific World Journal
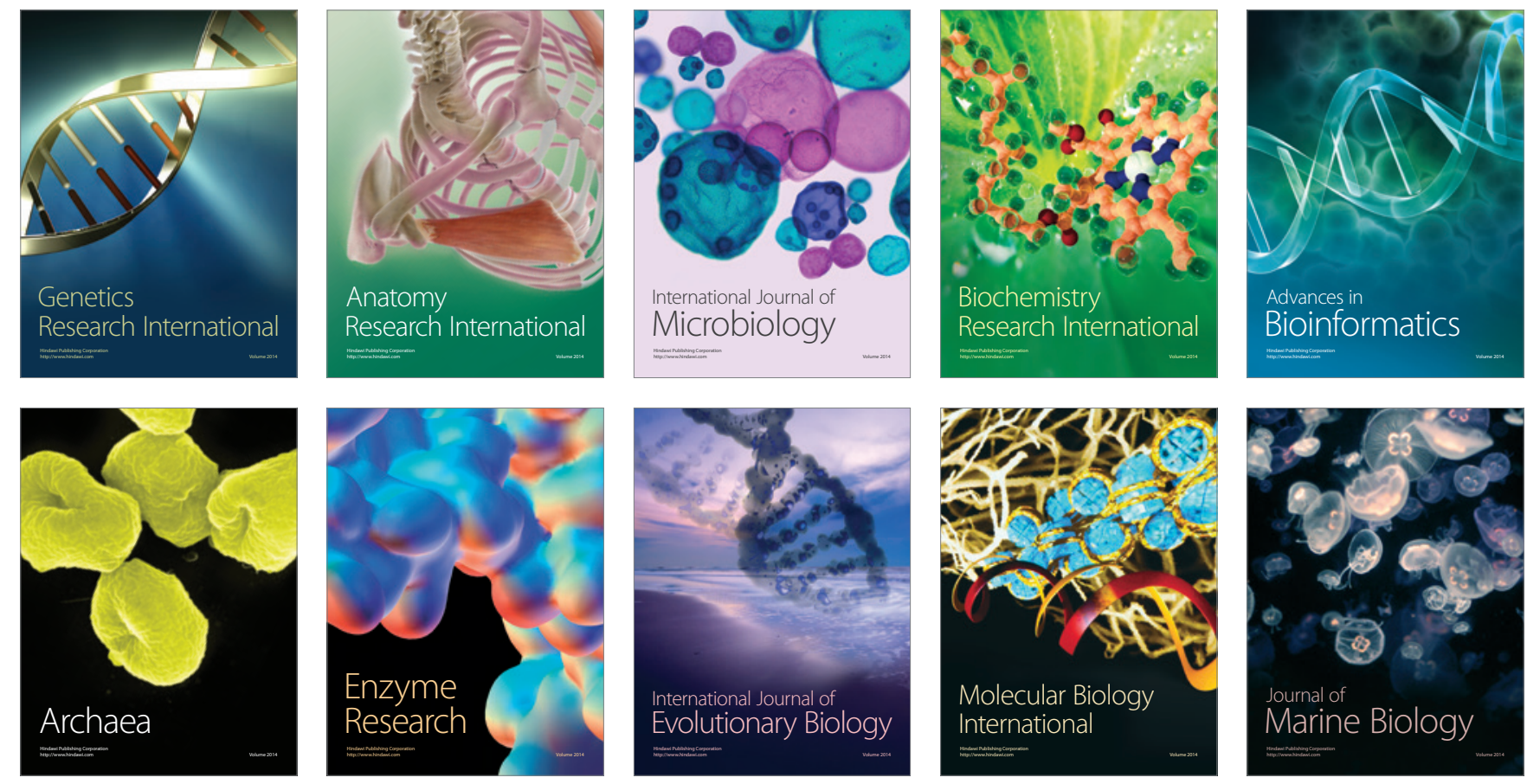